\title{
Effects of Carbon Monoxide and Cyanide Poisoning On Human Health
}

\section{Thomas NA and Jaiswal A*}

Department of Epidemiology and Public Health, Central University of Tamil Nadu, Thiruvarur, Tamil Nadu, India

*Corresponding author: Ajeet Jaiswal, Department of Epidemiology and Public Health, Central University of Tamil Nadu, India, Email: rpgajeet@gmail.com

\section{Research Article}

Volume 5 Issue 1

Received Date: March 29, 2021

Published Date: May 07, 2021

DOI: $10.23880 /$ phoa- 16000182

\section{Abstract}

Carbon monoxide and Cyanide are most familiar poisonings. Fire accidents act as main source for both poisonings. Carbon monoxide poisoning is formed from incomplete combustion of carbon compounds. Cyanide is readily available chemical and widely used in many industries like jewelry, paper, textiles. It is also used as weapon by terrorists. The methodology used in this paper is the review of secondary data sources available on the subject from library, internet, PubMed. Works done by several experts in the field was collected and interpreted. This review aims at detailed study on effects of carbon monoxide and cyanide poisoning. Both poisonings have almost similar kind of effects on human body and the effect of poisoning on body varies according to the dose. The review highlights that there is a need of effective and accurate antidote for both poisonings. The preventive measures can reduce the number and lethality from the exposures and psychiatric referral is essential for patients with intentional poisoning.

Keywords: Carbon Monoxide; Cyanide; Carboxyheamoglobin; Oxyheamoglobin; Hydroxycobalamine; HBOT; Hypoxia

Abbreviation: MPO: Myeloperoxidase; ROS: Reactive Oxygen Species.

\section{Introduction}

Carbon monoxide poisoning and Cyanide poisoning are the two most known types of intoxication. Both can be occurred individually as well as together. Presences of both poisonings are seen in fire accidents [1]. These types of intoxications can be accidental or intentional [2]. Since Greek and Roman periods we are aware on dangerous effects of carbon monoxide. It was used for executions earlier [3]. It is a gas without color, taste and smell [4]. Incomplete combustion of carbon substances causes carbon monoxide poisoning [5].
The common sources of the toxicant are improper working of heating appliances, fires, improper ventilation of motor vehicles, generators, stoves, improper gas appliances. It can cause chronic and acute poisonings [5].

In United States there are approximately 40000 cases of CO poisoning. The fatality rate is 0.5 to 1 per 1000000 persons. Almost $50 \%$ of the fatal poisonings are due to carbon monoxide. It is seen as a major reason for death in fire accidents [2]. The carboxyl heamoglobin levels are seen high in three quarters of burnt patients, which indicates high probability of carbon monoxide poisoning [4]. Carbon monoxide poisoning goes undiagnosed in most burnt cases [6]. In Britain CO poisoning occur by central heating fault 
where as in U.S the main reason is through exhaust fumes [3].

History shows that in 1857 Claude Bernard found out that carboxyl heamoglobin is formed in CO poisoning by reversible displacement of oxygen from heamoglobin. In 1926 another fact on hypoxia was revealed, it was that hypoxia is not only caused by difficulty in oxygen transport but also due to poor tissue uptake of oxygen. In the years 1960-70s coal gas was converted to Carbon monoxide free natural gases. It reduced the number of poisonings. Similarly the poisoning from car exhausts was reduced by use of catalytic converters [3].

Carbon monoxide poisoning is seen severe in pregnant women. Due to carbon monoxide poisoning maternal mortality is between $19-24 \%$, while fetal mortality is $36-$ $67 \%$. It is the third most cause of accidental deaths in U.S [6]. Like carbon monoxide Cyanide is also a dangerous chemical. Cyanide poisoning is creating more morbidity now [6]. In 1786, cyanide was first isolated from Prussian blue dye as hydrogen cyanide. In 1800 it was extracted from almonds. Cyanide exists in various forms. It can be a gas as Hydrogen Cyanide and it can be a salt, Potassium Cyanide or sodium cyanide [7]. In low temperature Hydrogen cyanide looks like a pale blue or colorless liquid, while in high temperature it become gas with bitter almond smell [8]. Cyanide can be naturally found in cassava, lima beans, almonds, and seeds of apricots, apples, peaches [8]. They are cyanogenic foods [9]. It is also involved in cigarette smoking. It is used in many industries like paper, textiles, plastics, electroplating, photographic solutions, metal cleaning. It is utilized in gold industry to separate gold from its ore. Similar to carbon monoxide, fire accidents results in cyanide poisonings also [8].

According to toxic exposure surveillance system, in the period of 1993-2003 there were 3165 cyanide poisonings.35\% of burnt patients have high levels of cyanide content in their blood, showing probability of cyanide poisonings [7]. Cyanide is used for intentional poisonings, both for murders and homicides. For murder attempts melamine, polyurethane, poly acrilonitrile, hydrogen cyanide are used [9]. It is also used by terrorists to pose threats. They pose threat mainly to international food and water supplies. Jonestown massacre is the most known incident of using cyanide as a weapon. More than 900 deaths occurred by drinking cyanide containing liquid. Osama Bin Ladan and ISIS also make threats by cyanide poisonings [10]. In World War 2 hydrogen cyanide was used by Germans as Zyklon B, as a genocidal agent (CDC). Drinking water was contaminated by industrial waste containing cyanides [9]. It acts as an ideal terrorist weapon as it is rapid in action, readily available, does not require any special knowledge to use. And it is able to cause mass confusion and difficulties to society. After all it require large amount of resources to recover from the attack [10]. Cyanide exposure is highly lethal to pregnant women. Both the mother and fetus are affected by cyanide poisoning [6]. As both carbon monoxide poisoning and cyanide poisoning can be formed in fire accidents, the treatment for one poisoning indirectly reduce other also. Physicians should take a special care to find out such poisonings in fire victims to avoid undiagnosed conditions [6].

\section{Methodology}

The present study is based on secondary resources available on the topic Carbon monoxide and cyanide poisoning. Data are collected from library, PubMed and internet sites concerned using the search terms carbon monoxide poisoning, cyanide poisoning, hyperbaric oxygen therapy. The search was done up to November 5 2020. Works done by several experts in the field are selected and further analysis and interpretation are done.

\section{Results and Discussion}

\section{Carbon Monoxide Poisoning}

Etiology: In carbon monoxide poisoning, heamoglobin binds to CO. Heamoglobin binds to carbon monoxide with an affinity 240 times greater than oxygen [5]. This association result in formation of carboxyheamoglobin [2]. It causes the leftward shift of oxygen-heamoglobin dissociation curve of oxyheamoglobin [3]. The binding of $\mathrm{CO}$ forms stabilization in heamoglobin. This stabilized form is known as R state. It increases affinity for oxygen in other sites of heamoglobin. It reduces the release of oxygen [4]. Carbon monoxide shows greater affinity to myoglobin compared to heamoglobin and cause myocardial infarction and hypotension. Both of these finally result in cellular hypoxia [3].

Similarly carbon monoxide binds to ferrous ion of Cytochrome oxidase of mitochondria and stop oxidative phosphorylation. It causes decrease in Adenosine triphosphate production or the energy production of body [10]. The shuffling of electrons continues to take place between components of electron transport chain and form superoxides, which can cause cell, damage [4].

Pathophysiology: Carbon monoxide enters into the body mainly through inhalation. It enters the lungs and diffuses to alveolar membranes and then into heamoglobin of blood [6]. Studies shows that inhalation of carbon monoxide causes more toxicity when compared to transfusion of similar concentration of carbon monoxide exposed RBC. In case of non smokers the carboxyheamoglobin levels greater than $2 \%$ and for smokers a level greater than $10 \%$ is enough to produce symptoms [4]. The carboxyheamoglobin level in 
blood of poisoned patient is directly correlated with clinical manifestation [3].

The presence of excess carbon monoxide can activate platelets by displacing NO from heamproteins in platelet. These NO react with super oxides and form peroxynitrate and inhibit function of mitochondria [4]. Thus carbon monoxide poisoning increase the activation of platelets [11]. These activated platelets stimulate neutrophils in blood to release myeloperoxidase (MPO). The release of MPO results in inflammatory effects by activating more neutrophils [12]. It again causes the formation of Xanthine oxidase which generates reactive oxygen species (ROS). Thus both MPO and ROS catalyze lipid peroxidation and increase the myelin content in body. This myelin increases the lymphocyte responses and activates microglia of brain [4]. Finally all these process leads to neurological and cardiac injuries [12].

Carbon monoxide poisoning causes inactivation of Calcium ATPase in plasma membrane and cause large influx of calcium ions, which results in brain injury. The intracellular lipases and proteases are activated by decrease in ATP production, which causes the depolarization of mitochondrial membranes and can cause death of the person [4]. Carbon monoxide exposure can increase carbon monoxide production in body [13]. The brain systolic heam levels are increased by CO exposure [4]. It induces activation of heamoxygenases, which is dependent on heam content of body [13]. Then the heamoxygenase enzyme form CO from tissues and increase the effects of poisoning.

Toxico Kinetics: According to World Health Organization, a long term exposure greater than $6 \mathrm{ppm}$ of carbon monoxide is toxic to human beings [4]. The half life of carbon monoxide is 320 minutes in a standard room with $21 \%$ of oxygen. While in $1000 \%$ of oxygen the half life reduces to less than 90 minutes. In hyperbaric oxygen condition of 3 ATM the half life of $\mathrm{CO}$ again reduces to 23 minutes so hyperbaric oxygen therapy is used for reducing the effects of carbon monoxide poisoning [2].

Clinical Manifestations: The signs and symptoms vary with concentration and duration of exposure. Low concentration of toxicant causes both cardiovascular and neurobehavioral effects. Acute and lengthy exposure leads to coma and death, the starting of chronic poisoning can be mistaken as flu depression, food poisoning [3]. The common clinical manifestations are headache, nausea, weakness, lethargy. Lethargy can lead to misdiagnosis of chronic fatigue syndrome [14]. Acute CO poisoning also show symptoms like tachycardia, hypertension, central nervous symptoms, abdominal pain, dizziness, paresis, convulsions, unconsciousness, myocardial ischemia, arterial fibrillation, pulmonary edema, erythrocytosis, hyperglycemia, muscle necrosis, acute renal failure, parkinsonism. Young adults express peripheral neuropathy also Psychiatric effects like irritability, depression and neuropsychological defects like impairment to attention, short term memories are also expressed [14,15]. Body organs like kidney, liver, pancreas are rarely affected. Muscle necrosis is caused by the increase in creatine phosphokinase. Chronic exposures in pregnant ladies cause growth retardation, fetal distress, death of fetus [3].

Diagnosis and Treatment: The diagnosis of carbon monoxide poisoning should be based on clinical triad, which assesses consistent symptoms, history of recent exposure, increased carboxyheamoglobin levels. Of these carboxy heamoglobin levels act as confirmation for the suspect [4]. But recent studies shows that normal levels of carboxy heamoglobin can't be taken as absence of carbon monoxide poisoning [4]. Pulse CO oximetry measurement is also used for diagnosis fetus, but the differentiation between wavelength of carboxy heamoglobin and oxyheamoglobin is difficult. Carbon monoxide breath analyzer is a simple screening test that can be used, but smoking and alcohol use of patients confound the result [3].

There are no available antidotes for the poisoning. 100\% normobaric oxygen therapy and hyper baric oxygen therapy are used and found effective. Both methods remove the carbon monoxide from heamoglobin by increasing partial pressure of oxygen, which in turn increase the dissociation rate [16]. Normobaric oxygen therapy decreases the half life into 74 minutes while HBOT decreases the half life into 20 minutes. HBOT shows a reversal effect on inflammation and dysfunction of mitochondria [4]. It is highly effective against neuro cognitive dysfunction. It is recommended for serious cases of acute poisonings [17].

Other than these, some other methods are used. Application of high concentration of oxygen with carbon dioxide is used as CO poisoning cause decrease in carbon dioxide level in body. So this method increases ventilation from carbon dioxide. Similarly normocapnic hyperpnea, extracorporeal membrane oxygenation, photo dynamic blood illuminations are used as optional methods. The study of Roderique and collegues claims that hydroxycobalamine and ascorbic acid can be used to convert $\mathrm{CO}$ into carbon dioxide. Cyclo dextrin encapsulated porphyrin complex are also found helpful by Kitagshi and colleagues [4].

The survivors have to compromise with quality of life with low cognitive performance, depression, stress [18]. In case of intentional poisoning they are referred to psychiatric counseling. Follow up of the patient is recommended within 4-8 weeks after exposure. Special care should be given to depression, neurocognitive impairments and myocardial 
infarction [2].

Prevention Measures: To find out CO poisoning CO detectors are installed in home. It should be on same level as fuel burning appliance. The alarm should be kept in a place, which is heard from all rooms. It minimizes the risk of exposure during sleep [5]. Public health campaigns should be done to make awareness on people about incidents on home and workplaces. The visible example of the campaign is shown by U.S through their "invisible killer campaign". The use of catalytic converters reduces the exposure from automobiles [4]. Public education should be provided by gas industry also. Physicians, engineers, gas industries should be well aware of the issue. Proper ventilation should be done in houses [3]. The person should be removed from the exposure immediately to prevent complications [2].

\section{Cyanide Poisoning}

Etiology: Cyanide is readily available and widely used chemical. It is well known for its toxic effects. Exposure to cyanide can be occurred through contaminated air, water, food, soil [8]. Exposure through inhalation depends on respiratory condition of individuals. Oral exposure results in continued absorption of toxicant [10].

Pathophysiology: Inhalation of cyanide gas is having more toxicant effects than ingestion. Cyanide diffuses into blood after exposure [7]. After absorption $\mathrm{CN}$ maintain equilibrium between cyanide anion and undissociated form of HCN. HCN form can easily cross cell membrane compare to other forms.HCN also inhibit enzymes like succinic dehydrogenase, mutase, and cytochrome oxidase of electron transport chain. Cyanide binds with ferric ion of cytochrome a3 in electron transport chain of mitochondria and inhibits oxidative phosphorylation and thereby aerobic respiration. Thus anaerobic metabolism increases and it results in academia and hyper lactemia. Potassium cyanide and sodium cyanide change to hydrogen cyanide in stomach acidic conditions [10]. Cyanide is a hepato toxic and neuro toxic chemical [6].

Toxic Kinetics: The concentration of $40 \mathrm{~mol} / \mathrm{L}$ is enough to express signs and symptoms of the poisoning. The body contains a special detoxification mechanism for cyanide. The enzyme named rhodanese help in this. It is found particularly in liver and muscle. Rhodanese convert cyanide to thiocyanate. Thiocyanate is soluble in water, so it is excreted through urine. Thiosulfate acts as a sulfur donor in this mechanism [7].

Clinical Manifestations: In exposures through inhalations symptoms are expressed within seconds. But in oral ingestion of poison it will take hours to express the symptoms. Symptoms vary with concentration of poison in body. The lethal dose of cyanide is $50 \mathrm{mg}$, an exposure greater than 50 mg cause death of individuals in 5-15 minutes. It will take at least 1-4 hours, if we are consuming small quantities of cyanide [19]. The low dose symptoms are headache, vomiting, dizziness, mild confusion; abdominal pain and high dose symptoms are hyperventilation, tachycardia, bradycardia, respiratory arrest, hypotension, dyspnea, seizures, coma and can cause death [10]. Increased lactate levels in blood and high concentration of oxygen levels in venous can cause sepsis, myocardial infarction, liver failure [10]. For patients in coma 2 clinical features are important. They are absence of cyanosis and bradycardia. Cyanide inhibits oxidative phosphorylation and thereby oxygen transport. So a fully oxygenated heamoglobin will be present and cyanosis is absent in them [19].

Diagnosis and Treatment: The toxic effect of poisoning is directly related to quantity of cyanide in body. The diagnostic methods include quantification of cyanide in blood, urine, saliva. But analysis of blood is taken as reliable. The reliability of oral swab depends on the quantity of cyanide in saliva at the time of swab collection. Whereas in case of urine, smoking increase cyanide concentration in urine and due to filtration of kidney the actual content of cyanide is not available. The diagnosis can be toxidrome, which is symptom based analysis of exposure. It helps to avoid delay in treatment by waiting laboratory confirmation [10].

The treatment methods are administration of $100 \%$ oxygen and decontamination methods like using activated charcoal, gastric lavage. The effective treatment is providing antidote intravenously or intraosseous. It should be done without delay. Intravenous administration requires skill. Treatment for cyanide poisoning can be divided into 3. First one is methamoglobin generators and nitric oxide donors. It includes sodium nitrite, amyl nitrite, and dimethyl aminophenol. They create hypotension and methemoglobinemia [10]. Use of sodium nitrite can cause cerebral haemorrhage [20]. The second group is sulfur donors. It includes sodium sulfate and glutathione [10]. Sodium thiosulfate is a category $\mathrm{C}$ drug for pregnant women. It create protection for fetus by creating a gradient towards mother [6]. The third group is directly binding agents, like hydroxycobalamine, cobalt ethylene diamine tetra- acetate [10]. Hydroxycobalamine is an expensive antidote. It should be given intravenous within 10-15 minutes of exposure [21]. Hydroxycobalamine convert cyanide into cyanocobalamine or vitamin B12 and it is excreted through urine. But it can cause kidney injury [6]. Cobalt EDTA creates a cobaltocyanide and reduces toxic effects [22]. In case of intentional poisonings they should be referred to psychiatric counseling [7].

Prevention Measures: As breathing cause high toxic effects avoid situations that lead to inhalation of the toxicants, 
especially in labs. Leave the area of exposure as soon as possible. Remove the clothing and discard the exposed clothes. Wash the exposed body parts with soap and water. Avoid touching contaminated areas, clothes, jewelry. Avoid mouth to mouth resuscitation as it cause risk to the person who provide CPR [8]. Provide education to public and workers about such possibilities [7].

\section{Discussion}

Carbon monoxide and cyanide are most common poisonings. Carbon monoxide poisonings are common in fire accidents [2]. It sometimes causes death of individuals before responding to it. For example carbon monoxide poisoning causes death of person in sleep before affected by the fire, in a fire accident. CO poisoning cause formation of carboxyhaemoglobin and affect cellular respiration of an individual. This condition of cellular hypoxia often leads to death [3]. Similar to carbon monoxide poisoning, cyanide poisoning also results from fire accidents. But it is most known as a suicidal drug or murder drug. It is also used as a chemical weapon by terrorists. It also affects cellular respiration and cause cellular hypoxia and death [10].

There are some similarities between these two intoxications. Carbon monoxide attaches to ferrous ion of heamoglobin while cyanide attaches to ferric ion of heamoglobin, both affect the oxidative phosphorylation. The symptoms for both poisonings are also same almost. Only the timing and severity is different in both. Similar type of treatments like hyperbaric oxygen therapy and hydroxycobalamine are used. So treatment of one poisoning indirectly reduce the other toxic effects [6].

There are no $100 \%$ accurate diagnosis techniques for both. There is a need for rapid, simple, accurate diagnostic measures. Similarly an effective antidote is absent for CO poisoning and completely safe and efficient antidote is absent for cyanide poisoning $[4,10]$. So research should be done in focus to these issues. Special care should be done for detection and treatment for poisoning in fetus. Physicians should take special considerations to avoid masking of carbon monoxide or cyanide poisonings in fire accidents [6]. Intentional poisonings should definitely refer to psychiatric department [2]. Public should aware on such possibilities and should take preventive measures to avoid poisonings.

\section{Conclusion}

Carbon monoxide and cyanide are highly toxic to human health. Carbon monoxide poisonings are common in fire accidents and it affects the aerobic respiration and ATP synthesis [2]. Cyanide also has similar effects on human body. Headache, dizziness, vomiting, nausea, coma are the common symptoms expressed in both [7]. Hyperbaric oxygen therapy is the effective treatment for carbon monoxide poisonings, while hydroxycobalamine is the effective antidote for cyanide poisonings [6]. The morbidity and mortality from these poisonings can be reduced by taking effective preventive measures.

\section{References}

1. Breen PH, Isserles SA, Westley J, Roizen MF, Taitelman UZ (1995) Combined carbon monoxide and cyanide poisoning: a place for treatment. Anesth Analg 80(4): 671-677.

2. Hanley ME, Patel PH (2020) Carbon Monoxide Toxicity. In: StatPearls [Internet]. Treasure Island (FL): StatPearls Publishing.

3. Blumenthal I (2001) Carbon monoxide poisoning. J R Soc Med 94(6): 270-272.

4. Rose JJ, Wang L, Xu Q McTiernan CF, Shiva S, et al. (2017) Carbon Monoxide Poisoning: Pathogenesis, Management, and Future Directions of Therapy. Am J Respir Crit Care Med 195(5): 596-606.

5. Wu PE, Juurlink DN (2014) Carbon monoxide poisoning. CMAJ 186(8): 611.

6. Culnan DM, Coffman CB, Bitz GH, Capek KD, Tu Y, et al. (2018) Carbon Monoxide and Cyanide Poisoning in the Burned Pregnant Patient: An Indication for Hyperbaric Oxygen Therapy. Ann plast surg 80(3S2): S106-S112.

7. Graham J, Traylor J (2020) Cyanide Toxicity. In: StatPearls [Internet]. Treasure Island (FL): StatPearls Publishing.

8. CDC (2018) Facts about Cyanide. Centers for Disease control and prevention.

9. Tshala-Katumbay DD, Ngombe NN, Okitundu D, David L, Westaway SK, et al. (2016) Cyanide and the human brain: perspectives from a model of food (cassava) poisoning. Ann N Y Acad Sci 1378(1): 50-57.

10. Hendry HTB, Ng PC, Witeof AE, Mahon SB, Brenner M, et al. (2019) A Review on Ingested Cyanide: Risks, Clinical Presentation, Diagnostics, and Treatment Challenges. J Med Toxicol 15(2): 128-133.

11. Karabacak M, Varol E, Turkdogan KA, Duman A, Akpinar 0 (2013) Mean Platelet Volume in Patients With Carbon Monoxide Poisoning. Angiology 65(3): 252-256.

12. Thom SR, Bhopale VM, Han ST, Clark JM, Hardy KR (2006) Intravascular neutrophil activation due to carbon monoxide poisoning. Am J Respir Crit Care Med 174(11): 
$1239-1248$.

13. Ryter SW, Choi AM (2009) Heme oxygenase-1/carbon monoxide: from metabolism to molecular therapy. Am J Respir Cell Mol Biol 41(3): 251-260.

14. Lader M, Morris R (2001) Carbon monoxide poisoning. J R Soc Med 94(10): 552.

15. Choi IS (2001) Carbon monoxide poisoning: systemic manifestations and complications. J Korean med sci 16(3): 253-261.

16. Casillas S, Galindo A, CamReyes LA, Varon J, Surani SR (2019) Effectiveness of Hyperbaric Oxygenation Versus Normobaric Oxygenation Therapy in Carbon Monoxide Poisoning: A Systematic Review. Cureus 11(10): e5916.

17. Buboltz JB, Robins M (2020) Hyperbaric Treatment of Carbon Monoxide Toxicity. In: StatPearls [Internet].
Treasure Island (FL): StatPearls Publishing.

18. Quinn DK, McGahee SM, Politte LC, Duncan GN, Cusin C, et al. (2009) Complications of carbon monoxide poisoning: a case discussion and review of the literature. Prim Care Companion J Clin Psychiatry 11(2): 74-79.

19. Lee JM, Bennett MA, Sherwell JM (1970) Cyanide selfpoisoning. Br Med J 4(5738): 780-781.

20. Bain JT, Knowles EL (1967) Successful treatment of cyanide poisoning. Br Med J 2(5554): 763.

21. Lavon O, Avrahami A, Eisenkraft A (2017) Effectiveness of isosorbide dinitrate in cyanide poisoning as a function of the administration timing. BMC pharmacology and toxicology 18(1): 13.

22. Thomas TA, Brooks JW (1970) Accidental cyanide poisoning. Anaesthesia 25(1): 110-114. 